placed jejunostomy (SJ). Direct percutaneous endoscopic jejunostomy (DPEJ) is increasingly used as an alternative to these modalities: Avoiding the intrinsic problems associated with the narrow calibre PEG-J and the tendency of displacement and retrograde migration; and is less invasive than SJ insertion, which also requires an enterotomy and enteropexy. Although progress with deep enteroscopy over the last decade has facilitated DPEJ placement, the presence of post-surgical intra-abdominal adhesive disease may still reduce success rates and procedure safety. In this setting, miniport laparoscopic-assisted DBE (lap-DBE) has the potential to provide safe and successful placement while maintaining the relatively minimally invasive approach of the endoscopic pull-through technique.

Methods Prospective assessment of outcomes of DPEJ placement by DBE and lap-DBE placed at our tertiary referral institution since June 2012.

Results 10 patients (6 [60\%] female, median age 40 years [range: $27-43$ years]) with chronic gastroparesis underwent DBE or lap-DBE facilitated DPEJ placement. Miniport laparoscopic assistance was only required in patients with a history of abdominal surgery (30\% [3/10]) and allowed us to identify and divide any underlying adhesions laparoscopically, facilitating DPEJ placement under direct endoscopic and laparoscopic vision, without the need for an enterotomy or surgical enteropexy. In this series DPEJ placement was successful in all 10 patients: Estimated depth of insertion [mean \pm SD] $66 \pm 12$ centimetres post-pylorus and procedure time [mean \pm SD] $49 \pm 114$ min. There were no immediate procedure-related complications and no delayed complications, morbidity or mortality at a mean follow-up of 339 days [range: 175-576 days].

Conclusion DPEJ placement by DBE is successful and safe. In patients with a history of abdominal surgery and underlying adhesive disease, lap-DBE should be considered, as it may enhance procedure success and safety.

Disclosure of Interest None Declared.

\section{PTU-057 THE "POOR MAN'S CELL-BLOCK" SAMPLE PREPARATION METHOD FOR EUS-FNA OF MEDIASTINAL AND RETROPERITONEAL LESIONS DOES NOT REQUIRE ATTENDING PATHOLOGY STAFF OR CYTOLOGY EXPERTISE}

${ }^{1}$ T Bracey*, 'JB King, ${ }^{2} \mathrm{D}$ Shetty, ${ }^{2} \mathrm{~B}$ Fox. 'Department of Cellular Pathology, Plymouth Hospitals NHS Trust, Plymouth, UK; ${ }^{2}$ Department of Radiology, Plymouth Hospitals NHS Trust, Plymouth, UK

\subsection{6/gutinl-2014-307263.131}

Introduction We present a novel technique of sample preparation for endoscopic ultrasound (EUS) that is simple, convenient and yields a high diagnostic success rate. EUS-guided fine needle aspirate (FNA) is increasingly used to obtain tissue in the mediastinum and retroperitoneum. Compared with surgical biopsy, EUS is minimally invasive and safe. The procedure, however is not without risk and can occasionally be poorly tolerated. It is therefore imperative that sampled tissue is optimally prepared. Ideally sample preparation should be simple without the need for an attending pathologist, and enable specific diagnosis and prognostics. The novel "poor man's cell block" (PMCB) technique, recently adopted in our institution for all EUS FNA, fulfils this need.

The PMCB technique allows the entire sample to be processed "as a biopsy". No slide preparation skills are needed and pathology staff need not be present. Special equipment or centrifugation is unnecessary, and samples can be reported without specific expertise or training in cytopathology. PCMB enables additional studies such as immunohistochemistry to enable subclassification and risk stratification of some neoplasms.

Methods All mediastinal and retroperitoneal histology/cytology reports since starting we started using the PMCB technique (2012-2013) were retrieved from the pathology database.

Results 23 mediastinal and 33 retroperitoneal reports were retrieved, of which 18 mediastinal and 27 retroperitoneal samples respectively were prepared with the PMCB technique.

All of the mediastinal PMCB samples (100\%) were diagnostic. Neoplasms were accurately subclassified, and several benign samples were corroborated by the presence of non-necrotising granulomas.

$63 \%$ of retroperitoneal PMCB samples were diagnostic. More specific diagnoses were afforded by the PMCB technique vs cytology (stromal and perineural invasion was seen in many pancreatic PMCB samples, enabling a "definitive" invasive diagnosis). In addition, a spindle cell GIST, and well differentiated endocrine carcinoma were diagnosed and both approximately graded/risk stratified.

Conclusion The PMCB technique is a simple, reliable and costeffective EUS-FNA sample preparation technique that in our hands appears superior to conventional cytology preparations (83\% diagnostic rate $\mathrm{PMCB}$ vs $57 \%$ cytology). We suggest PCMB can be reported by pathologists without cytology training/expertise. PMCB allows more accurate diagnosis with the additional benefit of immunohistochemistry allowing more accurate diagnosis and risk stratification for some neoplasms.

\section{REFERENCE}

Mayall, F, Darlington, A. The poor man's cell block. J Clin Pathol 63:837-838.

Disclosure of Interest None Declared.

\section{PTU-058 MACHINE LEARNING CREATES A SIMPLE ENDOSCOPIC CLASSIFICATION SYSTEM FOR DETECTING DYSPLASIA IN BARRETT'S OESOPHAGUS WITH I-SCAN IMAGING AND OPENS THE WAY TO STANDARDISED TRAINING AND ASSESSMENT OF COMPETENCE}

${ }^{1} V$ Sehgal*, ${ }^{2}$ A Rosenfeld, ${ }^{1} D G$ Graham, ${ }^{1} M R$ Banks, ${ }^{1} \mathrm{RJ}$ Haidry, ${ }^{1} \mathrm{LB}$ Lovat. ${ }^{1}$ Gastroenterology, University College London Hospital (UCLH), London, UK; ${ }^{2}$ Industrial Engineering, Jerusalem College of Technology, Jerusalem, Israel

\subsection{6/gutjnl-2014-307263.132}

Introduction Barrett's oesophagus (BE) is the pre-cursor for oesophageal adenocarcinoma. Endoscopic surveillance is performed to detect dysplasia in BE as it is likely to be amenable to curative treatment. Current surveillance relies on white-light endoscopy to obtain 4-quadrant biopsies through every $2 \mathrm{~cm}$ of the $\mathrm{BE}$ segment. This samples less than $5 \%$ of the BE epithelium and is likely to miss dysplasia.

A novel endoscopic image enhancement technology, i-Scan (PENTAX HOYA, Japan), has been developed to improve lesion recognition in the gastrointestinal tract (GIT). i-Scan uses postprocessing light filtering to provide real-time analysis and enhancement of the mucosa and microvasculature.

We evaluated the accuracy of i-Scan using a mucosal $(\mathrm{M})$ and vascular (V) classification system for BE amongst 3 expert (consultant) endoscopists. Machine learning (ML) generates simple rules, known as a decision tree, to improve dysplasia detection and validate our classification system. To our knowledge, ML has never been applied for dysplasia detection in the GIT. 